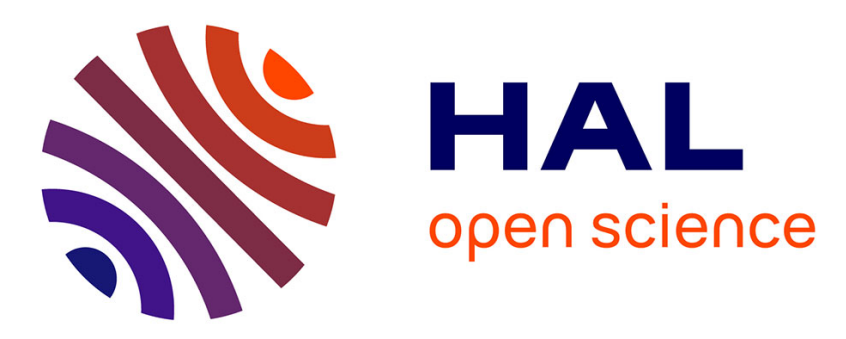

\title{
La production de théorie comme opération de hiérarchisation de concepts. Principes et applications aux sciences de gestion
}

\author{
Matthieu Mandard
}

\section{- To cite this version:}

Matthieu Mandard. La production de théorie comme opération de hiérarchisation de concepts. Principes et applications aux sciences de gestion. Gérer et Comprendre. Annales des Mines, 2021, 1 (143), pp.49-56. 10.3917/geco1.143.0049 . halshs-02983024

\section{HAL Id: halshs-02983024 \\ https://shs.hal.science/halshs-02983024}

Submitted on 8 Mar 2021

HAL is a multi-disciplinary open access archive for the deposit and dissemination of scientific research documents, whether they are published or not. The documents may come from teaching and research institutions in France or abroad, or from public or private research centers.
L'archive ouverte pluridisciplinaire HAL, est destinée au dépôt et à la diffusion de documents scientifiques de niveau recherche, publiés ou non, émanant des établissements d'enseignement et de recherche français ou étrangers, des laboratoires publics ou privés. 


\title{
La production de théorie comme opération de hiérarchisation de concepts
}

\section{Principes et applications aux sciences de gestion ${ }^{1}$}

\author{
Matthieu Mandard ${ }^{1}$ \\ CREM - Centre de recherche en économie et management, UMR CNRS 6211
}

Résumé : Que signifie produire de la théorie ? Si les diverses méthodes de recherche ont reçu une attention considérable dans la littérature scientifique, la nature même de la production théorique qui en résulte est assez largement passée sous silence. L'objectif de cet article est donc d'éclaircir ce sujet en montrant, à partir de travaux issus des sciences de gestion, que la production de théorie est une opération de hiérarchisation de concepts, dans la mesure où les différentes modalités de raisonnement scientifique conduisent à différentes opérations de hiérarchisation de concepts.

Mots clés : concepts, épistémologie, raisonnement scientifique, relations sémantiques hiérarchiques, théorie

1 Nous tenons à remercier Philippe Robert-Demontrond pour les commentaires apportés suite à la relecture d'une version préliminaire de l'article. 
L'objectif clef du travail scientifique est de produire de la théorie, c'est-à-dire d'établir des relations entre des concepts (Corley et Gioia, 2011). Afin de s'assurer de la qualité de cette production, les diverses méthodes de recherche ont reçu une attention considérable, et notamment en sciences de gestion, où de très nombreux articles ont été écrits sur le sujet : comment identifier un gap dans la littérature, comment élaborer et mettre en œuvre un protocole d'investigation, ou comment évaluer la qualité de la contribution proposée (Shepherd et Suddaby, 2017).

Malgré cette abondante littérature, une question reste en suspens : qu'en est-il de la production théorique obtenue? Ou, pour être plus précis, quelles sont les mises en relation de concepts qui résultent du travail scientifique ? Chacun sait bien entendu que l'on attend d'une contribution théorique qu'elle soit originale, utile, ou plus complète que les explications existantes. Mais cela ne nous renseigne pas tellement sur la nature même des théories scientifiques, qui est toujours en débat (Shepherd et Suddaby, 2017 ; Suddaby, 2014).

Le problème est que, en raison de cette relative incompréhension, la portée des différentes méthodes de recherche est régulièrement débattue, notamment entre tenants des travaux hypothético-déductifs et partisans des études de terrain, chacun vantant les mérites respectifs de son approche et rejetant - sinon méprisant - les productions de l'autre camp. Quelle est la nature de la production théorique obtenue lorsque l'on teste des hypothèses sur un échantillon représentatif ? Quelle est celle qui résulte d'une enquête qualitative ? En quoi se distinguent-elles ? Quelles sont les autres modalités de production de théorie ? Et pour quels résultats ?

L'objectif de cet article est d'éclaircir ce sujet en montrant, à partir de travaux issus des sciences de gestion, que la production de théorie est une opération de hiérarchisation de concepts, dans la mesure où les différentes modalités de raisonnement scientifique conduisent à différentes opérations de hiérarchisation de concepts. Plus précisément, dans cet article, nous présentons tout d'abord les quatre principaux types d'opérations de hiérarchisation de concepts (particularisation, généralisation, partition et totalisation). Nous montrons ensuite que ces opérations s'avèrent être le produit des quatre principales manières de raisonner (déduction, induction, abduction et invention - la dernière étant régulièrement oubliée). Nous discutons finalement les implications de cette analyse, en montrant que ces opérations de hiérarchisation de concepts se complètent en un processus récursif.

Ce faisant, cet article peut constituer une analyse utile à tous ceux qui, chercheurs ou praticiens, souhaitent affiner leur compréhension du travail scientifique. II permet également de mettre en évidence le fait que les différentes modalités de raisonnement scientifique sont complémentaires et non pas substituables, et de rappeler que les oppositions récurrentes qui existent entre elles n'ont en réalité pas lieu d'être.

\section{Les opérations de hiérarchisation de concepts}


En linguistique, l'étude des relations sémantiques s'attache à identifier la nature des liens que des termes entretiennent. Ces relations entre concepts peuvent être hiérarchiques ou d'équivalence, selon que les termes sont ou non de même rang (Lehmann et Martin-Berthet, 2013). Dans le premier cas, les termes entretiennent un rapport d'inclusion - ainsi, OISEAU ${ }^{2}$ est un terme général qui englobe celui d'HIRONDELLE. Dans le second, les termes entretiennent un rapport de synonymie ou d'antonymie JEUNE est l'opposé de VIEUX.

C'est aux opérations de regroupement de concepts réalisées au travers de l'établissement de relations hiérarchiques que nous nous intéressons ici, dans la mesure où elles permettent d'organiser les termes en sous-systèmes, contrairement aux relations d'équivalence, qui ont pour seul but d'identifier la similarité ou la dissimilarité des termes d'un même rang. Ces regroupements de termes en sous-systèmes se divisent à leur tour en deux catégories : les relations général-particulier et les relations partie-tout (Tableau 1).

Tableau 1 - Les opérations de hiérarchisation de concepts

Type de relation sémantique hiérarchique

Relation généralparticulier

Relation partietout

\begin{tabular}{llll}
$\begin{array}{l}\text { Rang } \\
\text { hiérarchique } \\
\text { envisagé }\end{array}$ & Rang supérieur & Généralisation & Totalisation \\
\cline { 2 - 4 } & Rang inférieur & Particularisation & Partition
\end{tabular}

\subsection{Le général et le particulier}

Les relations général-particulier - également appelées relations genre-espèce organisent les liens entre des termes désignant les cas généraux, appelés hyperonymes, et des termes désignant les cas particuliers, appelés hyponymes (Lehmann et Martin-Berthet, 2013). II s'agit de relations logiques, car l'appartenance d'un cas particulier à un cas général s'établit mécaniquement à partir de leurs définitions respectives. Une VACHE ou un COCHON (hyponymes) sont ainsi des cas particuliers du genre MAMMIFĖRE (hyperonyme) en raison de leurs propriétés respectives.

Pour prendre des concepts usuels en sciences de gestion, ORGANISATION est un terme général qui englobe les termes ENTREPRISE ou ASSOCIATION, chacun de ces termes

2 Par souci de clarté, et comme cela est parfois d'usage en linguistique, les concepts analysés seront rédigés en lettres majuscules. 
désignant des cas particuliers d'organisations. De la même manière, RÉMUNÉRATION est un terme général qui regroupe les termes SALAIRE ou TRAITEMENT, chacun d'eux désignant également un type de rémunération particulier.

II convient de noter que ce type de mise en relation de concepts est à la fois multiple et évolutif (Lehmann et Martin-Berthet, 2013). Multiple, car un même terme peut avoir plusieurs hyperonymes en fonction du contexte. Le BREVET peut ainsi être classifié comme MÉCANISME DE PROTECTION DES INNOVATIONS, de même que le SECRET INDUSTRIEL, mais il peut également être répertorié comme VECTEUR DE RÉPUTATION au même titre qu'une PUBLICITÉ, dans la mesure où il permet de renseigner les parties prenantes d'une organisation quant au savoir-faire de celle-ci. Évolutif, car ces classifications ne sont pas figées. Ainsi, le brevet était historiquement envisagé à l'aune de son rôle de protection des innovations ; ce n'est qu'avec l'apparition d'une économie de l'innovation que son usage en tant que vecteur de réputation s'est développé et qu'il a alors également été classifié en tant que tel (Mandard, 2018).

\subsection{Le tout et les parties}

Les relations partie-tout organisent les liens entre des termes désignant les totalités, appelés holonymes, et des termes désignant les parties, qui sont appelés méronymes (Lehmann et Martin-Berthet, 2013). II s'agit ici de relations ontologiques, car le recensement des parties renseigne sur la nature même du tout. Une TASSE (holonyme) est ainsi composée d'un RÉCIPIENT et d'une ANSE (méronymes).

En sciences de gestion, une ENTREPRISE se subdivise ainsi en diverses fonctions: LOGISTIQUE, PRODUCTION, COMMERCIALISATION, etc. De la même manière, une RÉMUNÉRATION peut se décomposer en une PART FIXE et en une PART VARIABLE.

Les relations partie-tout sont généralement considérées comme étant de six principaux types (Winston, Chaffin et Herrmann, 1987) ${ }^{3}$ : composant-assemblage (e.g., boulon et machine), membre-ensemble (e.g., joueur et équipe), portion-masse (e.g., part et tarte), substance-objet (e.g., ciment et mur), phase-activité (e.g., développement et innovation), lieu-zone (Bretagne et France).

\section{Opérations de hiérarchisation de concepts et modalités de raisonnement associées}

Comment les opérations de hiérarchisation de concepts sont-elles réalisées ? C'est tout l'objet du travail scientifique, qui vise à assurer cette hiérarchisation au moyen de diverses modalités de raisonnement. Nous montrons ici que les quatre principaux types d'opérations de hiérarchisation de concepts s'avèrent en effet liés aux quatre principales modalités de raisonnement existantes que sont la déduction, l'induction, l'abduction et l'invention - ces éléments sont résumés dans le Tableau 2 ci-après.

3 D'autres classifications existent, mais il s'agit là de la plus populaire. 
II convient de noter que, l'objectif de cette section étant uniquement de montrer le lien qui existe entre modes de raisonnement et opérations de hiérarchisation de concepts, nous nous limiterons à une brève présentation de chacun de ces modes de raisonnement et renvoyons le lecteur aux références indiquées pour plus de détails quant à leur portée et quant à leurs limites.

Tableau 2 - Opérations de hiérarchisation de concepts et modalités de raisonnement associées

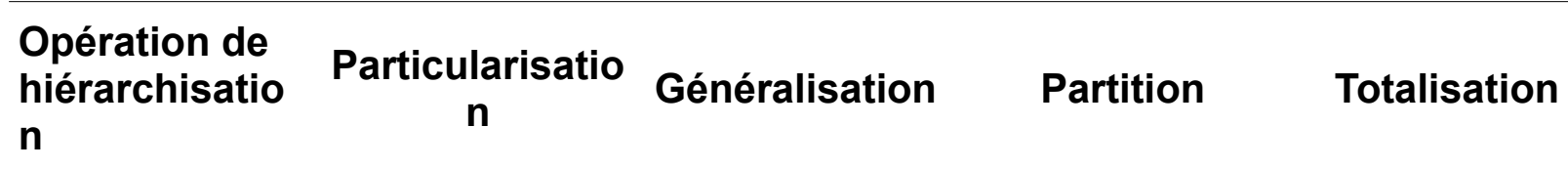

$\begin{array}{lcccc}\text { Principe de } & \begin{array}{c}\text { Caractériser le } \\ \text { cas particulier }\end{array} & \begin{array}{c}\text { Préciser le cas } \\ \text { général }\end{array} & \begin{array}{c}\text { Inventorier les } \\ \text { parties }\end{array} & \begin{array}{c}\text { Désigner le } \\ \text { tout }\end{array}\end{array}$

Concept à $\quad$ Hyponyme Hyperonyme $\quad$ Méronyme Holonyme
l'étude

Modalité de raisonnement Déduction Induction Abduction Invention associée

$\begin{array}{llll}\begin{array}{l}\text { Validité de la } \\ \text { relation établie }\end{array} \text { Prouvée } & \text { Probable } \quad \text { Plausible } & \text { Postulée }\end{array}$

\subsection{La particularisation comme produit du raisonnement déductif}

La particularisation est une opération de hiérarchisation de concepts qui consiste à attribuer à un cas particulier - l'hyponyme - les propriétés dont est doté un cas général. Ceci correspond typiquement au raisonnement déductif, qui part (1) d'une règle générale et (2) d'une explication pour en inférer une conclusion portant sur (3) un cas particulier (Peirce, 1878). À titre d'exemple, si (1) l'on part de la règle générale selon laquelle « les organisations sont dotées de la propriété A », et que (2) l'on dispose de l'explication "les associations sont des organisations ", alors (3) on peut en déduire que « les associations sont dotées de la propriété $A$ ». En d'autres termes, on attribue au cas particulier des associations la propriété A qui est celle des organisations en général.

Ce mode de raisonnement permet d'aboutir à une mise en relation de concepts prouvée - c'est-à-dire établie de manière irréfutable - dès lors que les prémisses sont valides 
(Mantere et Ketokivi, 2013). C'est le mode de raisonnement que l'on retrouve à titre principal dans tous les travaux qui procèdent par hypothèse - i.e., selon une démarche hypothético-déductive ${ }^{4}$.

S'agissant là du mode de raisonnement scientifique dominant, les exemples abondent. Dans un article consacré aux décisions d'achat, partant de (1) la règle générale selon laquelle "les décisions d'achat sont reportées en cas de manque d'informations " et de (2) l'explication " les décisions d'achat des produits de luxe sont des décisions d'achat ", Ladwein et Sánchez (2018) testent empiriquement l'hypothèse selon laquelle (3) « les décisions d'achat de produits de luxe sont reportées en cas de manque d'informations ». En vérifiant cette hypothèse, ils valident le transfert au cas particulier de la DÉCISION D'ACHAT DES PRODUITS DE LUXE d'une propriété dont est dotée la DÉCISION D'ACHAT en général. De la même manière, dans un article consacré aux conflits de rôle, partant de (1) la règle générale selon laquelle " les conflits de rôle chez les individus engendrent du stress " et de (2) l'explication "les conflits de rôle chez les cadres de santé sont des conflits de rôle chez les individus ", Rivière (2019) vérifie empiriquement I'hypothèse selon laquelle (3) « le conflit de rôle chez les cadres de santé engendre du stress ». Ici, elle valide le transfert au cas particulier du CONFLIT DE RÔLE CHEZ LES CADRES DE SANTÉ d'une propriété dont est doté le CONFLIT DE RÔLE CHEZ LES INDIVIDUS en général.

Quel que soit l'exemple choisi, le principe est toujours le même : il s'agit de mettre en évidence la manifestation particulière d'une règle générale. C'est le mode de raisonnement scientifique qui constitue la norme en sciences de gestion comme dans la plupart des autres champs scientifiques, même s'il nous faut souligner que notre discipline est plutôt ouverte à une pluralité de mode de raisonnements - et s'ouvre d'ailleurs de plus en plus -, et même si l'on peut également noter que nous bénéficions en Europe d'une ouverture épistémologique plus grande qu'en Amérique du Nord (Thiétart, 2014).

\subsection{La généralisation comme produit du raisonnement inductif}

La généralisation est une opération qui consiste à attribuer à un cas général l'hyperonyme - les propriétés dont est doté un cas particulier. II s'agit ici d'un raisonnement de type inductif, qui part quant à lui (3) d'un cas particulier et (2) d'une explication pour en inférer une conclusion portant sur (1) une règle générale (Peirce, 1878). En l'occurrence, si (3) si l'on part du cas particulier « les associations sont dotées

4 La mise en œuvre de ce mode de raisonnement n'est cependant pas sans poser deux principales difficultés (Mantere et Ketokivi, 2013). La première tient à la formulation du raisonnement. Un raisonnement déductif est bien souvent - et notamment en sciences de gestion - énoncé en langage naturel, ordinaire (par opposition au langage formel). Or, le langage ordinaire n'est pas exempt d'ambiguïtés, de connotations, qui en amoindrissent la cohérence logique. La seconde difficulté tient à la confrontation du raisonnement à l'expérience. D'un point de vue épistémologique, un énoncé théorique ne peut jamais être véritablement confirmé ou réfuté par une expérience empirique singulière. Lorsqu'une expérience contredit une théorie, il est en effet toujours possible de considérer que le résultat obtenu correspond à un cas qui échappe à la théorie, et d'amender cette dernière par des hypothèses ad hoc. 
de la propriété $A$ », et que (2) l'on dispose de l'explication "les associations sont des organisations ", alors (1) on peut en induire que "les organisations sont dotées de la propriété $A »$. En d'autres termes, on attribue au cas général des organisations la propriété A qui est celle des associations en particulier.

Contrairement au raisonnement déductif, ce mode de raisonnement permet seulement d'aboutir à une conclusion probable (Mantere et Ketokivi, 2013). L'observation de cas particuliers permet seulement de réaliser des extrapolations quant à la règle, qui ne peut être valide de manière absolue. C'est le mode de raisonnement employé par les travaux qui procèdent par étude de cas pour en inférer des conclusions d'ordre général ${ }^{5}$.

II s'agit là aussi d'un mode de raisonnement très répandu en sciences de gestion. À titre d'exemple, dans un célèbre ouvrage consacré aux cultures nationales, partant de (3) l'observation particulière selon laquelle «les cultures d'entreprise françaises, néerlandaises et étasuniennes sont respectivement marquées par une logique de l'honneur, du consensus et du contrat» et de (2) l'explication "la culture des entreprises est la culture des nations ", d'Iribarne (1989) en induit (1) la règle générale selon laquelle « les cultures des nations françaises, néerlandaises et étasuniennes sont respectivement marquées par une logique de l'honneur, du consensus et du contrat ". De la sorte, il transfère au cas général de la CULTURE DES NATIONS des propriétés qui sont celles de la CULTURE DES ENTREPRISES en particulier. De manière similaire, dans un article consacré aux fablabs, partant de (3) l'observation particulière selon laquelle « le fablab de Renault favorise l'ambidextrie organisationnelle » et de (2) l'explication " le fablab de Renault est le fablab d'une organisation ", Lô (2017) en induit (1) la règle générale selon laquelle "les fablabs des organisations favorisent l'ambidextrie organisationnelle ». Ici, il transfère au cas général des FABLABS DES ORGANISATIONS une propriété qui est celle du FABLAB DE RENAULT en particulier.

Ce mode de raisonnement gagne en popularité depuis quelques décennies dans notre discipline en raison de l'essor des réflexions méthodologiques qui permettent sa mise en œuvre. Parmi les principales contributions sur le sujet figure bien entendu la grounded theory, élaborée par Glaser et Strauss à partir des années 1960 (Glaser et Strauss, 1967), mais aussi la démarche formulée par Kathleen Eisenhardt dans son article "Building theories from case study research » publié à la fin des années 1980 (Eisenhardt, 1989), ou encore, plus récemment, la méthodologie proposée par Dennis Gioia (Gioia, Corley et Hamilton, 2013).

5 De la même manière que pour la déduction, la mise en œuvre du raisonnement inductif comporte deux types de difficultés essentielles, constitutives du fameux problème de l'induction (Ketokivi et Mantere, 2010). D'une part, puisque toute observation n'est jamais qu'observation du passé, on ne peut jamais être véritablement certain de la capacité prédictive de la règle générale proposée : ce qui a toujours été ne sera pas forcément demain. Ceci est d'autant plus vrai en sciences sociales, où les acteurs sont susceptibles de modifier leur comportement lorsqu'ils prennent connaissance des lois qui le régissent. D'autre part, puisque toute observation n'est jamais qu'observation d'événements particuliers auxquels l'observateur confère une signification particulière, la généralisation proposée peut toujours être remise en question. Un même ensemble de données peut en effet toujours donner lieu à des généralisations alternatives - c'est la thèse de la sous-détermination de la théorie par l'expérience. 


\subsection{La partition comme produit du raisonnement abductif}

La partition est une opération de hiérarchisation de concepts qui consiste à identifier les parties - les méronymes - d'un tout. Cette opération est typiquement la résultante d'un raisonnement abductif, qui vise à proposer (2) une explication qui met en relation (1) une règle générale et (3) un cas particulier (Peirce, 1878). Pour reprendre notre exemple association / organisation, ce mode de raisonnement vise à proposer (2) l'explication "les associations sont des organisations » qui met en relation (1) la règle générale selon laquelle " les organisations sont dotées des propriétés $A, B$ et $C$ » et (3) le cas particulier selon lequel « les associations sont dotées des propriétés $A, B$ et $C$ ». Ici, on établit donc que les associations sont dotées des mêmes propriétés $A, B$ et $C$ que les organisations ${ }^{6}$.

Ni prouvée, ni probable, la conclusion est ici de l'ordre du plausible pour autant que le raisonnement proposé soit vraisemblable - c'est-à-dire pour autant que les concepts à l'étude semblent bien disposer de certaines propriétés communes (Mantere et Ketokivi, 2013). C'est le mode de raisonnement qui est employé lorsque l'on souhaite éclairer des concepts dont les propriétés sont mal comprises ${ }^{7}$.

Moins répandu que les deux précédents car plus délicat à mettre en œuvre, ce mode de raisonnement est à l'origine de nombreuses contributions théoriques majeures. Ouchi a par exemple proposé l'explication selon laquelle "les organisations sont des clans ", pour mettre en évidence le fait que les ORGANISATIONS et les CLANS peuvent avoir en commun de fonctionner selon des principes de SOLIDARITÉ, de TRADITIONS, de CROYANCES et de VALEURS COMMUNES (Ouchi, 1980). Rousseau a élaboré sa théorie du contrat psychologique à partir de l'explication selon laquelle " les attentes d'un individu envers une organisation sont un contrat ». Elle met ainsi en évidence le fait que les ATTENTES D'UN INDIVIDU ENVERS UNE ORGANISATION comme les CONTRATS peuvent représenter un SYSTĖME D'OBLIGATIONS MUTUELLES (Rousseau, 1989). Et il en est ainsi de tous nos grands cadres théoriques (Ketokivi, Mantere et Cornelissen, 2017) : la théorie des coûts de transaction établit ainsi que les ORGANISATIONS sont des STRUCTURES DE GOUVERNANCE, la théorie de l'agence dit que les ORGANISATIONS sont des NOEUDS DE CONTRATS, la théorie de la citoyenneté organisationnelle énonce que les EMPLOYÉS sont des CITOYENS, etc. II s'agit à chaque fois d'établir le fait que les concepts cibles disposent de propriétés similaires aux propriétés des concepts sources avec lesquels ils sont mis en rapport.

II s'agit d'un mode de raisonnement qui conduit ultérieurement à mener des programmes de recherche, dans la mesure où la relation conceptuelle nouvellement

6 Pour être tout à fait précis, l'explication « les associations sont des organisations » est une analogie qui conduit à doter les associations des mêmes propriétés que les organisations, c'est-à-dire à procéder à une abduction. Ce que formule Umberto Eco en ces termes $(1993,182)$ : " en anglais abduction signifie "rapt ", "enlèvement " (...). Si j'ai un résultat curieux dans un champs de phénomènes non encore étudié (...), je dois aller " enlever » ou " emprunter » une loi ailleurs. Si vous voulez, je dois raisonner par analogie. "..

7 Toute la difficulté du raisonnement abductif consiste à identifier l'existence d'une similarité - d'une analogie - entre des concepts cibles mal compris et des concepts sources bien connus, pour ensuite attribuer aux premiers les propriétés des seconds - pour ensuite procéder à l'abduction. 
établie est ensuite amenée à être vérifiée et amendée au travers de raisonnements déductifs ou inductifs. Le domaine de validité de cette relation conceptuelle se trouve ainsi précisé et les concepts à l'étude finalement mieux compris.

\subsection{La totalisation comme produit du raisonnement inventif}

Dernière opération de hiérarchisation de concepts, la totalisation consiste à rassembler une somme de parties en un tout - en un holonyme. II s'agit d'un acte d'invention qui met en relation des propriétés afin de proposer un concept nouveau.

La totalisation est une opération de hiérarchisation de concepts couramment pratiquée, de manière intuitive, lorsque nous vivons une expérience nouvelle. Par exemple, lorsque nous sommes affectés par une maladie que nous ne parvenons pas à identifier, nous disons souvent que nous avons QUELQUE CHOSE, ce qui nous permet de rassembler différents symptômes tels qu'une CÉPHALÉE, de la TEMPÉRATURE ou des MAUX DE VENTRE. Lorsque nous voyons un objet qui nous est inconnu, nous le qualifions de TRUC, ce qui nous permet par exemple de désigner un objet GRIS, CARRÉ, avec des BOUTONS. Au niveau sociétal, de la même manière, des néologismes apparaissent régulièrement pour décrire de nouveaux phénomènes sociaux. II en est ainsi du néologisme INFOX récemment institué, qui désigne une INFORMATION qui est FAUSSE, ou du néologisme SELFIE, qui désigne un AUTOPORTRAIT PHOTOGRAPHIQUE.

C'est aussi ce qui est fait dans le champ scientifique, lorsque l'on souhaite identifier un phénomène jusqu'alors ignoré (Nersessian, 2010). II s'agit même d'une opération sans laquelle aucune abduction, induction ou déduction ne serait possible. La mise en œuvre de ces raisonnements nécessite en effet de disposer au préalable d'une règle générale telle que "les organisations sont dotées des propriétés $A$, B, et $C$ ", susceptible d'être mise en rapport avec des cas particuliers. Cette démarche inventive est de l'ordre du postulat. II s'agit d'une proposition de définition qui devra ensuite être mise à l'épreuve des modes de raisonnement précédemment présentés afin d'en tester la validité et éventuellement de la corriger.

Régulièrement oublié, ce type de raisonnement fait donc partie intégrante du travail scientifique (Nersessian, 2010). De nombreux termes sont ainsi apparus au cours de l'histoire des sciences, tels que ATOME, ÉLECTRON, GĖNE ou NEURONE (Thagard et Shelley, 1997). En biologie par exemple, le concept de SÉLECTION NATURELLE est proposé par Charles Darwin en 1859 afin de désigner la VARIATION, I'ADAPTATION et l'HÉRÉDITÉ des caractéristiques biologiques des membres d'une espèce. De manière similaire, le concept de SYSTĖME SOLAIRE prend son essor au $16^{e}$ siècle avec les travaux de Nicolas Copernic afin de désigner un système planétaire composé du SOLEIL et de divers objets célestes qui gravitent autour : TERRE, LUNE, SATURNE, etc.

En sciences de gestion, de nouveaux concepts apparaissent également de manière régulière, et ce notamment en raison du fait qu'il s'agit d'une discipline encore récente et 
en plein développement ${ }^{8}$. Le concept de FORCE CONCURRENTIELLE a par exemple été proposé dans les années 1970 par Michael Porter afin d'identifier les acteurs susceptibles d'altérer la capacité des organisations à faire du profit, tels que les CLIENTS, les SUBSTITUTS, ou les FOURNISSEURS (Porter, 1979). De la même manière, le terme de CAPACITÉS DYNAMIQUES a été élaboré à partir des années 1990 afin de désigner l'aptitude des organisations à ADAPTER, INTÉGRER puis RECONFIGURER leurs compétences dans un environnement économique sans cesse changeant (Teece et Pisano, 1994) ${ }^{9}$. Et les autres exemples en la matière abondent: HYPERCOMPÉTITION, SOCIOMATÉRIALITÉ, AMBIDEXTRIE ORGANISATIONNELLE, STUPIDITÉ FONCTIONNELLE, etc.

Quelles sont les conditions d'apparition d'un nouveau concept ? Trois principaux critères doivent être remplis (Gerring, 1999) ${ }^{10}$. II faut tout d'abord que ce concept englobe une combinaison nouvelle de propriétés - de méronymes - : il ne doit pas déjà exister de concepts équivalent. II faut également que ce concept rassemble une combinaison de propriétés stable dans le temps : ce concept doit saisir des régularités empiriques. II faut enfin que le concept proposé soit utile à l'approfondissement de notre compréhension du monde : il doit combler un manque conceptuel. C'est précisément ce que font Teece et Pisano dans leur article consacré à l'introduction du concept de CAPACITÉs DYNAMIQUES (1994): ils s'attachent à défendre l'idée selon laquelle ce concept décrit une réalité ignorée des appareillages conceptuels antérieurs ; ils montrent que ADAPTER, INTÉGRER puis RECONFIGURER ses compétences sont les problématiques que rencontre toute organisation dans un environnement toujours en mutation; ils défendent enfin la nécessité du recours à ce concept pour comprendre le fonctionnement des organisations.

\section{Discussion: les opérations de hiérarchisation de concepts, un processus récursif}

Si les différentes manières de raisonner et les opérations de hiérarchisation de concepts auxquels elles conduisent ont été présentées de manière séparée, il s'avère qu'elles se complètent en un processus récursif, dans la mesure où chaque opération de hiérarchisation de concepts prend appui sur les autres opérations (voir Figure 1).

8 II faut cependant prendre garde à la prolifération conceptuelle à laquelle on assiste dans notre discipline, car l'introduction de nouveaux concepts semble parfois non pas tant viser à désigner de nouveaux phénomènes qu'à procurer une rente de situation à leurs auteurs (Mehrpouya et Willmott, 2018).

9 Cette définition fut ultérieurement revue par Teece, qui proposa par la suite de désigner par CAPACITÉS DYNAMIQUES l'aptitude des organisations à IDENTIFIER et à ÉVALUER les opportunités et les menaces, puis à se TRANSFORMER en conséquence (Teece, 2007).

10 D'autres critères existent, notamment en ce qui concerne le caractère esthétique du concept proposé, mais nous ne présenterons ici que les critères qui nous semblent les plus décisifs (pour approfondir cette question, voir Gerring, 1999). 
Figure 1 - Les opérations de hiérarchisation de concepts, un processus récursif

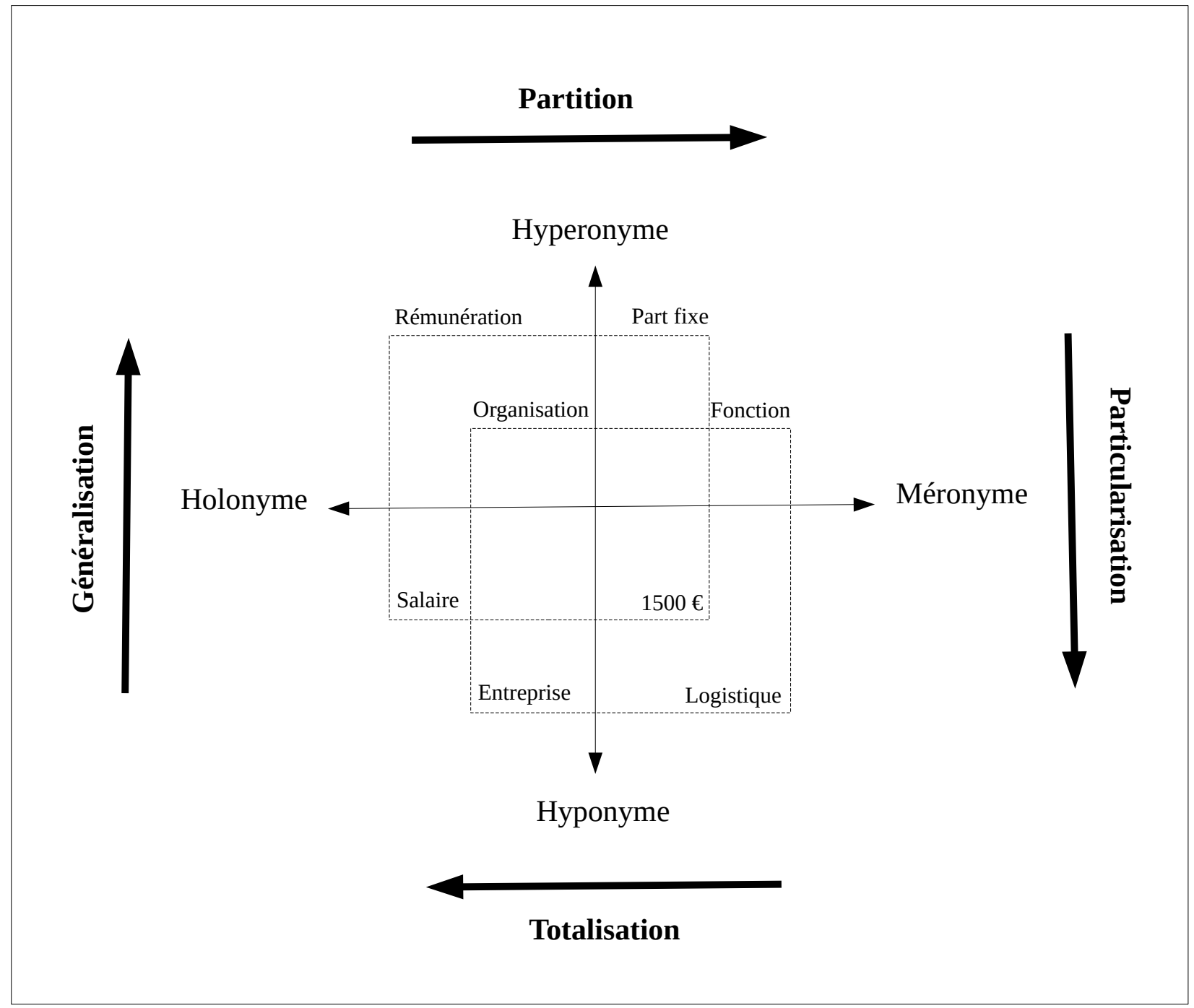

D'un point de vue synchronique, les différentes opérations de hiérarchisation de concepts apparaissent en effet complémentaires. Un SALAIRE est ainsi un type de RÉMUNÉRATION (hyperonyme) en même temps qu'il est composé de différents éléments tels qu'une PART FIXE (méronyme). De la même manière, la LOGISTIQUE est à la fois un type de FONCTION et une partie d'une ENTREPRISE. En conséquence de quoi, loin de se substituer, raisonnements inductif, déductif, abductif et inventif se complètent afin d'assurer la mise en relation de concepts, chacun possédant un gradient de validité qui lui est propre.

Par ailleurs, d'un point de vue diachronique, en raison de la récursivité du processus, la redéfinition de concepts ou l'inclusion de nouveaux concepts vient nécessairement questionner ceux existants (Thagard, 1990). Ainsi, le concept de SYSTĖME SOLAIRE (holonyme) s'est élargi au gré des nouvelles observations pour intégrer des objets 
célestes tels que SATURNE, URANUS ou NEPTUNE (méronymes), évolutions qui ont amené à reconsidérer les comportements des astres appartenant à ce système. De la même manière, depuis le début des années 1960, en sciences de gestion, le champ de la STRATÉGIE a ainsi progressivement évolué pour intégrer dans son périmètre les concepts de RESSOURCES, de COMPÉTITION ou de PARTIES PRENANTES. Ces évolutions ont conduit à l'apparition de nouveaux sujets de recherche en même temps qu'elles ont conduit à reconsidérer les concepts initialement identifiés comme étant au cœur de la stratégie, tels que ceux d'ACTION ou d'ENVIRONNEMENT (Ronda-Pupo et Guerras-Martin, 2012).

\section{Conclusion: de la nécessité d'un pluralisme en matière de raisonnement scientifique}

En montrant que les quatre principales modalités de raisonnement scientifique conduisent respectivement à quatre types d'opérations de hiérarchisation de concepts différents, et en soulignant le fait que ces opérations se complètent en un processus récursif, cet article propose une clarification de la nature des productions théoriques. Cet faisant, il nous semble qu'il offre une analyse utile à tous ceux qui, chercheurs ou praticiens, souhaitent affiner leur compréhension du travail scientifique.

Dans le même temps, ce travail constitue également un plaidoyer pour un pluralisme en matière de raisonnement scientifique. En raison de la complémentarité des diverses opérations de hiérarchisation de concepts, les oppositions qui existent entre modalités de raisonnement n'ont en réalité pas lieu d'être ; nous profitons donc de cet article pour rappeler qu'il serait néfaste de restreindre le travail scientifique à l'une ou l'autre modalité. II convient en particulier de prendre garde au tropisme déductiviste maintes fois dénoncé. Au risque de cantonner la recherche scientifique à des opérations de particularisation - à des opérations de détermination des espèces en lesquelles se déclinent un terme. Au risque donc d'empêcher le renouvellement des connaissances produites, au travers de l'identification des cas généraux dans lesquels s'inscrivent des termes (opération de généralisation) comme au travers de la mise en évidence de nouvelles relations parties-tout (opérations de partition et de totalisation). 


\section{Bibliographie}

CORLEY K. \& GIOIA D. (2011), " Building theory about theory building: what constitutes a theoretical contribution? ", Academy of management review, vol. 36, n 1, pp. 12-32.

ECO U. (1993), De superman au surhomme, Grasset.

EISENHARDT K. (1989), "Building theories from case study research ", Academy of management review, vol. $14, \mathrm{n}^{\circ} 4$, pp. 532-550.

GERRING J. (1999), "What makes a concept good? A criterial framework for understanding concept formation in the social sciences ", Polity, vol. 31, $n^{\circ} 3$, pp. 357393.

GIOIA D., CORLEY K. \& HAMILTON A. (2013), "Seeking qualitative rigor in inductive research: Notes on the Gioia methodology ", Organizational research methods, vol. 16, $\mathrm{n}^{\circ} 1$, pp. 15-31.

GLASER B. \& STRAUSS A. (1967), Discovery of grounded theory: Strategies for qualitative research, Hawthorne, NY, Aldine de Gmyter.

IRIBARNE P. D' (1989), La logique de l'honneur: gestion des entreprises et traditions nationales, Seuil.

KETOKIVI M. \& MANTERE S. (2010), "Two strategies for inductive reasoning in organizational research ", Academy of Management Review, vol. 35, n 2, pp. 315-333.

KETOKIVI M., MANTERE S. \& CORNELISSEN J. (2017), " Reasoning by analogy and the progress of theory ", Academy of Management Review, vol. 42, n 4, pp. 637-658.

LADWEIN R. \& SÁNCHEZ A. (2018), " L'ambivalence dans la décision d'acheter des produits de luxe: le cas des millennials ", Management Avenir, vol. , n 8, pp. 155-178.

LEHMANN A. \& MARTIN-BERTHET F. (2013), Lexicologie: Sémantique, morphologie et lexicographie, Armand Colin.

LÔ A. (2017), « Un FabLab d'entreprise pour favoriser l'ambidextrie des salariés - Étude de cas chez Renault », Revue française de gestion, vol. 43, n² 264, pp. 81-99.

MANDARD M. (2018), "Les usages du brevet: vers une définition du brevet comme mécanisme de gouvernance des activités d'innovation des organisations ", Innovations, vol. 3, n 57 , pp. 165-187.

MANTERE S. \& KETOKIVI M. (2013), " Reasoning in organization science ", Academy of Management Review, vol. 38, n 1, pp. 70-89.

MEHRPOUYA A. \& WILLMOTT H. (2018), "Making a Niche: The Marketization of Management Research and the Rise of 'Knowledge Branding' ", Journal of Management Studies, vol. 55, $n^{\circ} 4$, pp. 728-734.

NERSESSIAN N. (2010), Creating scientific concepts, MIT press. 
OUCHI W. (1980), "Markets, bureaucracies, and clans", Administrative science quarterly, vol. $25, \mathrm{n}^{\circ} 1$, pp. 129-141.

PEIRCE C. (1878), "Deduction, induction, and hypothesis ", Popular science monthly, vol. 13, p. 470-482.

PORTER M. (1979), "The five competitive forces that shape strategy ", Harvard business review, vol. 86, $\mathrm{n}^{\circ}$ Mars-Avril, pp. 137-145.

RIVIÈRE A. (2019), "Tensions de rôle et stress professionnel chez les cadres de santé à l'hôpital public-L'effet modérateur des stratégies d'ajustement ", Revue française de gestion, vol. 45, $\mathrm{n}^{\circ} 282$, pp. 11-35.

RONDA-PUPO G. \& GUERRAS-MARTIN L. (2012), " Dynamics of the evolution of the strategy concept 1962-2008: a co-word analysis ", Strategic Management Journal, vol. $33, n^{\circ} 2$, pp. $162-188$.

ROUSSEAU D. (1989), "Psychological and implied contracts in organizations", Employee responsibilities and rights journal, vol. 2, $\mathrm{n}^{\circ}$ 2, pp. 121-139.

SHEPHERD D. \& SUDDABY R. (2017), " Theory building: A review and integration ", Journal of Management, vol. 43, n 1, pp. 59-86.

SUDDABY R. (2014), "Editor's comments: Why theory? ", Academy of Management Review, vol. 39, $\mathrm{n}^{\circ}$ 4, pp. 407-411.

TEECE D. (2007), " Explicating dynamic capabilities: the nature and microfoundations of (sustainable) enterprise performance ", Strategic management journal, vol. 28, $n^{\circ} 13$, pp. 1319-1350.

TEECE D. \& PISANO G. (1994), " The dynamic capabilities of firms: an introduction ", Industrial and corporate change, vol. 3, $\mathrm{n}^{\circ} 3$, pp. 537-556.

THAGARD P. (1990), " Concepts and conceptual change ", Synthese, vol. 82, n² 2, pp. 255-274.

THAGARD P. \& SHELLEY C. (1997), "Abductive reasoning: Logic, visual thinking, and coherence ", Logic and scientific methods, Springer, pp. 413-427.

THIÉTART R.-A. (2014), Méthodes de recherche en management - 4ème édition, Dunod.

WINSTON M., CHAFFIN R. \& HERRMANN D. (1987), "A taxonomy of part-whole relations ", Cognitive science, vol. 11, n 4, pp. 417-444. 